\title{
Mantenimiento y cambio de tópico en un corpus de ELE
}

\author{
Kim Collewaert, An Vande Casteele \\ Vrije Universiteit Brussel
}

\begin{abstract}
The present investigation studies clean topic-continuity and topic-shift in a corpus of oral narratives produced by Dutch-speaking learners of Spanish as a foreign language. It thereby investigates to what extent the learners use the null pronoun, the explicit personal pronoun and the proper name in order to mark both topic-continuity and topic-shift, considering the different referential options in Dutch and Spanish, respectively non-pro-drop and pro-drop languages. Embedded in the cognitive linguistics tradition, the study is primarily based upon the Accessibility Theory (Ariel 1990) and the accessibility scales created by Ariel and Figueras Solanilla (2002). The corpus data were annotated in Excel and in the UAM CorpusTool.
\end{abstract}

Keywords: Spanish as a foreign language, Corpus linguistics, Discourse analysis, Anaphora resolution, Information Structure

\section{Introducción}

En el presente estudio se pretende investigar las nociones de 'mantenimiento de tópico ${ }^{1}$, (topic-continuity) y 'cambio de tópico' (topic-shift) en un corpus de narraciones orales producidas por aprendices neerlandófonos del español como lengua extranjera. La investigación se inscribe en una línea de estudios cognitivos, ya que la Teoría de la Accesibilidad de Ariel (1990) es la premisa principal del estudio. En el siguiente apartado, se presentarán las ideas principales de esta teoría, además de profundizar en las diferencias entre el sistema referencial del neerlandés y del español. Luego, se discutirán el corpus, que fue compilado en la Vrije

\footnotetext{
${ }^{1}$ Cf. Lambrecht (1994) para la definición de «tópico»: «the topic of the sentence is the thing which the proposition expressed by the sentence is about». De acuerdo con Lambrecht, distinguimos entre las nociones de «sujeto» $\mathrm{y}$ «tópico»: en la oración «a Beatriz le gusta Álvaro» analizamos el objeto indirecto «Beatriz/le» como tópico en vez del sujeto sintáctico «Álvaro».
} 
Universiteit Brussel, y la metodología empleada al coleccionar las narraciones y al etiquetar los parámetros estudiados en los programas de análisis Excel y UAM CorpusTool. Finalmente, proporcionaremos los primeros resultados con respecto al mantenimiento y cambio de tópico en nuestro corpus de aprendices del español como lengua extranjera.

\section{Marco teórico}

Los estudios cognitivos que se centran en la estructura informativa y en el fenómeno de la referencia afirman, en general, que la elección de una expresión referencial depende de su prominencia (saliency) en el discurso y de su givenness (Chafe 1976; 1996, Gundel et al. 1993, Prince 1981). En la Teoría de Accesibilidad, Ariel (1990) sostiene que la selección de una expresión referencial se basa en el grado de accesibilidad del referente, es decir, la medida en que es identificable para el destinatario, según las suposiciones del hablante. Esta elección depende, pues, del estatus cognitivo del referente, es decir de las asunciones del emisor sobre la representación mental del referente que tiene el receptor. Así, la introducción de un nuevo referente se correlaciona con el uso de expresiones largas, de alta carga informativa, como los sintagmas nominales. $\mathrm{Al}$ contrario, el mantenimiento de la referencia se realiza por medio de expresiones cortas, como el pronombre personal. Ariel también presentó una escala de accesibilidad - de alcance para el inglés -, en que se marca el grado de accesibilidad de las expresiones referenciales (Figura 1). En la adaptación al español elaborada por Figueras Solanilla (2002; Figura 2), destaca la adición del pronombre nulo, un recurso típico del español, ya que es una lengua pro-drop, al contrario que el inglés.

La oposición entre lenguas pro-drop y non-pro-drop también está presente en nuestra investigación, ya que el neerlandés, la lengua materna de nuestros participantes, también es una lengua non-pro-drop. Significa que, al marcar el mantenimiento de tópico, se debe utilizar un pronombre personal explícito (salvo en oraciones coordinadas, donde se permite el uso del pronombre nulo), y así se opone al español en que el pronombre nulo es la opción no marcada.

En lo que atañe al cambio de tópico, suele marcarse en español por el uso del pronombre personal explícito (Lozano 2009, 2016, 2018; Lubbers Quesada \& Blackwell 2009; Alonso-Ovalle et al. 2002; Luján 1999; Porto Dapena 1986; inter alia), mientras que en neerlandés se requiere adicionalmente un énfasis prosódico en el mismo pronombre, puesto que no es suficiente utilizar el pronombre personal explícito, que también es obligatorio en casos de mantenimiento de tópico. 
Most inaccessible topic

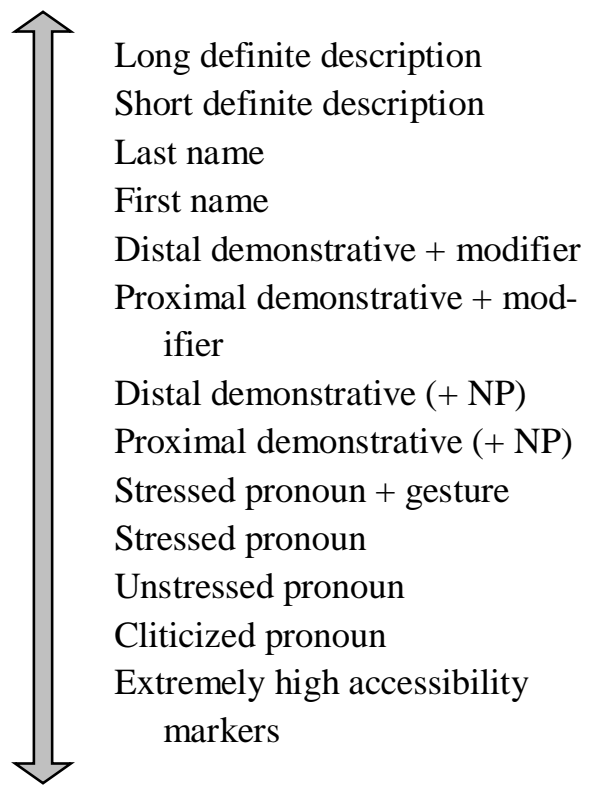

Most accessible topic

Figura 1. Escala de accesibilidad (Ariel 1990) Solanilla 2002)

\section{Most inaccessible topic}

Nominal phrases with definite arti-
cle
Proper nouns
Proper noun + non-restrictive
complement
Definite article + long modifier
Definite article + short modifier
Complete proper noun
$2^{\text {nd }}$ proper noun (surname)
$1^{\text {st }}$ proper noun (first name)
Demonstrative pronouns
Modified by full noun phrase
Independent demonstrative pro-
noun
Contrastive personal pronouns
Cliticized pronouns
Zero anaphora

Most accessible topic

Figura 2. Escala de accesibilidad (Figueras

La adquisición del funcionamiento del pronombre nulo y del pronombre personal explícito en español ya ha sido el sujeto de varios estudios, como, por ejemplo, Lubbers Quesada \& Blackwell (2009), Liceras \& Díaz (1999), Rothman (2009), Alonso-Ovalle et al. (2002) y Perales \& Portillo (2007). Cabe observar, no obstante, que en el presente estudio, también tenemos en cuenta el nombre propio como expresión referencial que puede utilizarse en casos de mantenimiento y cambio de tópico. Al nombre propio, como apunta Lozano (2016), siempre se le ha asignado el papel de introducir un nuevo referente en el discurso, mientras que se ha ignorado que también se utiliza a menudo en casos de cambio de tópico. Esta idea también se refleja en la escala de accesibilidad, donde el nombre propio 
se marca como una expresión referencial de baja accesibilidad, las que suelen utilizarse para la introducción de nuevos referentes ${ }^{2}$.

Por fin, cabe mencionar que existen varios factores que pueden afectar o influir en la accesibilidad de un referente. Distinguimos cuatro factores de influencia, a saber, la distancia entre una referencia y su antecedente, la presencia de competidores - es decir, otros referentes que intervienen entre dos referencias consecutivas a una entidad -, el cambio de una escena a otra, y por fin, la función sintáctica que ocupa el referente. En futuras investigaciones, se estudiará en qué medida influyen en la elección de las expresiones referenciales halladas en nuestro corpus.

\section{Hipótesis, corpus y metodología}

De acuerdo con los usos de los pronombres en neerlandés y en español, expuestos en el apartado anterior, se puede plantear como primera hipótesis si los aprendices neerlandófonos del español como lengua extranjera se mostrarán redundantes ${ }^{3}$ al marcar el mantenimiento de tópico y recurrirán a expresiones referenciales como el pronombre personal explícito o el nombre propio, ya que en neerlandés se debe utilizar un pronombre personal explícito en la mayoría de los casos de mantenimiento de tópico. Asismismo, se espera que los aprendices sean sobre-explícitos (overexplicit) marcando el cambio de tópico, con el empleo de un nombre propio, por ejemplo, porque todavía no han asimilado completamente el funcionamiento del español.

A fin de verificar estas hipótesis se compilaron dos corpora transversales. Por un lado, se recogieron datos de nativos neerlandófonos, que estudiaron español como lengua extranjera en la Vrije Universiteit Brussel, entre 2013 y 2017. Realizaron una prueba oral en el marco de una asignatura del segundo año del Grado en Lingüística y Estudios literarios - Opción Español, teniendo en aquel momento un nivel A2 de español. Por otro lado, participó en la tarea un grupo de nativos españoles, que forman el grupo de control de este estudio. La mayoría de los participantes también estudiaron en la Vrije Universiteit Brussel, en el marco del programa Erasmus+ o del Multilingual Master in Linguistics and Literary Studies.

\footnotetext{
${ }^{2}$ Cabe mencionar que en nuestro estudio distinguimos entre la nueva introducción de una entidad y el cambio de tópico, que es una función que puede atribuirse a referentes ya introducidos en el discurso anteriormente, al igual que la función de «mantenimiento de tópico».

${ }^{3}$ El término «redundante» (cf. Lozano 2016, 2018) se utiliza como sinónimo de «sobre-explícito» (overexplicit), mientras que la etiqueta de «sub-explícito» concuerda con la idea de ambigüedad.
} 
Los dos corpora, de aprendices y de nativos, cuentan con 36 y 21 textos respectivamente. A continuación, presentaremos algunos resultados provenientes del primer corpus, es decir, del corpus de los aprendices de ELE.

Los participantes realizaron una tarea de recuento basada en la telenovela española Yo soy Bea. En la compilación de ocho fragmentos de la serie aparecen seis personajes principales: Beatriz, Álvaro, Gonzalo, Diego, Cayetana y Francisco. El objetivo del estudio es investigar las referencias consecutivas que utilizan los participantes para referir a cada uno de estos personajes. La tarea misma consiste en dos fases consecutivas. Primero, los participantes miran el vídeo con el sonido puesto. Luego, lo miran otra vez, pero sin sonido, ya que el objetivo consiste en contar, con sus propias palabras, lo que están viendo. No se admite citar a los personajes o resumir la historia general. Después de la transcripción de los datos, se codificaron varios parámetros en Excel y en el UAM CorpusTool. El UAM CorpusTool (O’Donnell 2008) es una herramienta que permite a investigadores crear su propio esquema de análisis y después aplicarlo a un corpus de tex$\operatorname{tos}^{4}$, que se puede subir al CorpusTool. Después de que el investigador ha etiquetado el corpus, se pueden consultar los resultados para cada etiqueta. El programa también realiza pruebas chi-square $\left(\chi^{2}\right)$, contrastando los resultados de dos grupos investigados, como, en nuestro caso, los grupos no nativos y nativos, y menciona el intervalo de confianza ${ }^{5}$. En el siguiente apartado, se presentarán algunos primeros resultados con respecto al mantenimiento y cambio de tópico en el corpus de aprendices.

\section{Resultados preliminares}

Para obtener los resultados nos centramos de forma exclusive en las nociones de mantenimiento y cambio de tópico, y más específicamente, en los casos «puros» que se hallan en el corpus. En otras palabras, nos centraremos en las ocurrencias en que se produce el mantenimiento o el cambio de un tópico singular ${ }^{6}$ y que aparece en posición sintáctica de sujeto. Se excluyen, pues, referentes coordina-

\footnotetext{
${ }^{4}$ Se permite aplicar un esquema a textos completos (por ejemplo, a fin de marcar si el texto pertenece al corpus de nativos o al corpus de aprendices) o a elementos concretos de un texto (por ejemplo, las expresiones referenciales en los textos).

${ }^{5} 90 \%$ (= justamente no significativo) - 95\% (= significativo) - $98 \%$ (= altamente significativo)

${ }^{6}$ Solo se incluyen referencias singulares consecutivas. Es decir, no se incluyen referencias singulares que siguen una referencia copulativa/plural (ej. Álvaro y Gonzalo > Álvaro, los dos hombres > Álvaro). El paso de referencias plurales a singulares también ha sido etiquetado en el corpus.
} 
dos copulativos (ej. Álvaro y Gonzalo) y plurales (ej. los dos hombres). Asimismo, no se tienen en cuenta construcciones como la siguiente: «y justo cuando [Beatriz] entra, se encuentra con el futuro jefe de la empresa que es Álvaro [...] y creo que a ella, le gusta a primera vista Álvaro» (N131401), ya que el mantenimiento del tópico se realiza aquí con una construcción dativa. En el UAM CorpusTool, se etiquetaron los rasgos mencionados anteriormente, es decir, el mantenimiento/cambio de tópico, la función sintáctica del referente (sujeto) y el número del referente (singular/copulativo/plural).

El corpus de los aprendices contiene 36 textos, como se ha mencionado anteriormente, en los que localizamos un total de 3280 expresiones referenciales. Cabe mencionar que la investigación global pretende investigar las cadenas referenciales completas producidas por los participantes, es decir, también se incluyen referencias en posición de objeto (directo, indirecto, oblicuo), que no se incluyen en los resultados presentados aquí. En el corpus registramos 589 ocurrencias del mantenimiento de tópico «puro».

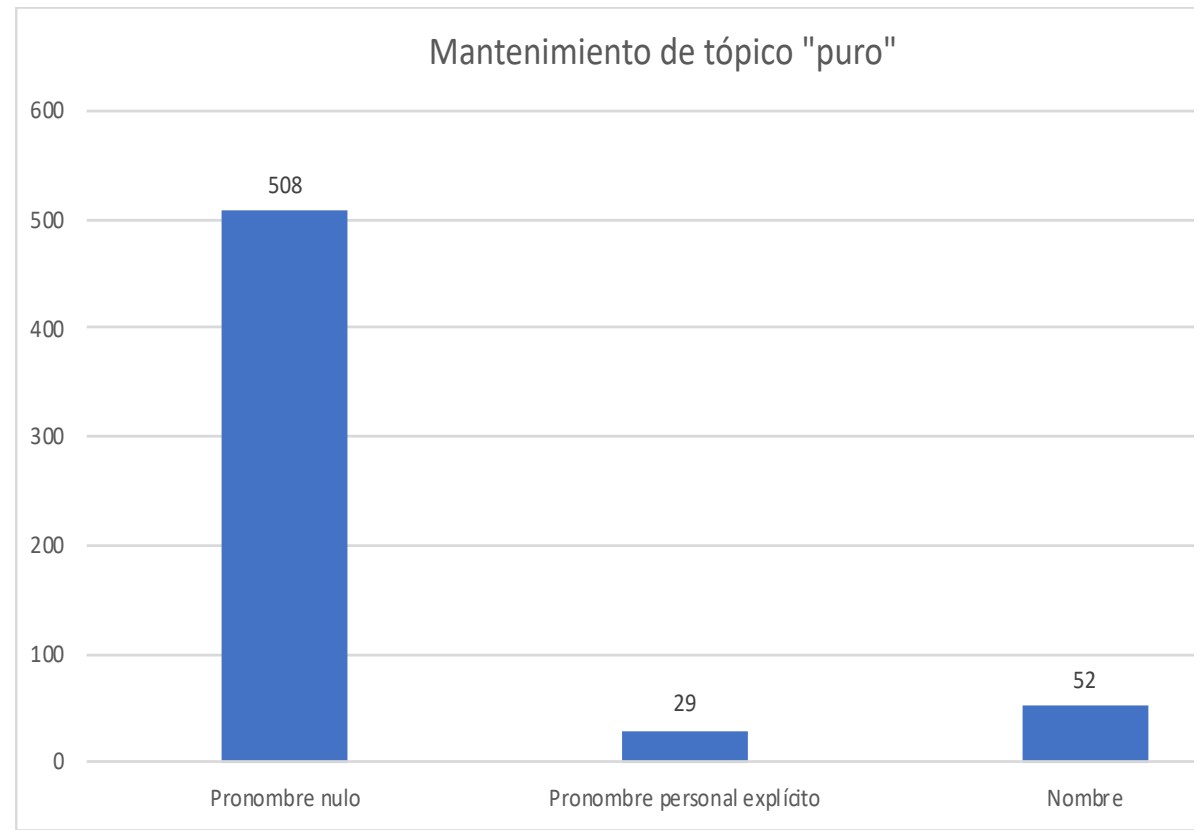

Figura 3. Mantenimiento de tópico «puro»

Como puede observarse en la figura (3), en la mayoría de los casos $(86,25 \%)$ se opta por un pronombre nulo, rasgo típico del español (Lozano 2009, 2016, 2018; 
Lubbers Quesada \& Blackwell 2009; Alonso-Ovalle et al. 2002; Luján 1999; Porto Dapena 1986; inter alia). Los aprendices, pues, demuestran que, a pesar de tener un nivel básico de español, ya tienen un buen dominio del uso del pronombre nulo, aunque su lengua materna no dispone de este recurso. Sin embargo, también se encuentran expresiones referenciales sobre-explícitas en el corpus, es decir, expresiones con más carga informativa de lo necesario para la identificación única del referente. Se trata de 29 pronombres personales explícitos y de 52 nombres, de los que 48 son nombres propios ${ }^{7}$. Se demuestra, pues, que el nombre propio puede desempeñar más papeles en el discurso que la función introductora que le suele ser asignado ( $c f$. Lozano 2016). En los ejemplos (1) a (3), se ilustra el mantenimiento de tópico con un pronombre nulo, un pronombre personal explícito y un nombre propio:

(1) y ella le está ignorando // no quiero no quiere hablar // está enfadada y y todavía no habla (NN1516207)

(2) y después uhm Beatriz no puede decir nada y ella es distraído un poco pienso (NN1617205)

(3) y Álvaro entre y uhm y Álvaro pregunta también qué se pasa (NN1415207)

En lo que concierne al cambio de tópico «puro», luego, se encuentran 301 ocurrencias en el corpus (Figura 4).

Solo en 45 casos (o sea, el 14,95\%), el cambio de tópico se realiza con el pronombre personal explícito, aunque este tipo de expresión referencial siempre se presenta como el marcador típico del cambio de tópico en español (Lozano 2009, 2016, 2018; Lubbers Quesada \& Blackwell 2009; Alonso-Ovalle et al. 2002; Luján 1999; Porto Dapena 1986; inter alia). Al contrario, cabe destacar el papel preponderante del nombre propio, que se utiliza en $195 \operatorname{casos}^{8}$ de cambio de tópico. Otra vez, pues, se demuestra que el nombre propio puede desempeñar otras funciones en el discurso que la introducción de nuevas entidades ${ }^{9}$, lo que hasta ahora, como mencionado antes, se ha ignorado en la mayoría de los estudios con respecto a la realización de referentes de 3 a persona del singular, que se focalizaban en la oposición entre el pronombre nulo y el pronombre personal explícito. Luego, destaca también el número considerable de pronombres nulos, que constituye el $18 \%$ de todos los casos de cambio de tópico. Recordamos que el pronombre nulo se utiliza para mantener la referencia a un tópico, por lo que, en general, su uso resulta ambiguo en casos de cambio de tópico. No obstante, antes

\footnotetext{
${ }^{7}$ Otros cuatro son sintagmas nominales (ej. el padre).

${ }^{8}$ Además de los 195 nombres propios, también se utilizan 7 sintagmas nominales.

${ }^{9}$ Repetimos que distinguimos entre las funciones de nueva introducción y cambio de tópico.
} 
de concluir que se trata de usos completamente ambiguos, se deberá verificar en qué medida intervienen indicios contextuales o semánticos en la identificación del referente. Los tres tipos de cambio de tópico - con el pronombre personal explícito, con el nombre propio y con el pronombre nulo - se ilustran en los ejemplos (4) a (6):

(4) pero no es lo que Beatriz quiere porque no quiere hablar con él // es un poco difícil porque sí no puede decir lo que ha entendido // pero él es amable con ella // y ella toma su distancia de él (NN1314206)

(5) Álvaro quiere que Beatriz dice algo pero Beatriz no dice nada // y Álvaro dice que quiere un médico para ella (NN1516202)

(6) y uhm Beatriz ha una herida uhm y un choc y uhm va a ayudar [= Álvaro] Beatriz con la herida un poco (NN1415204)

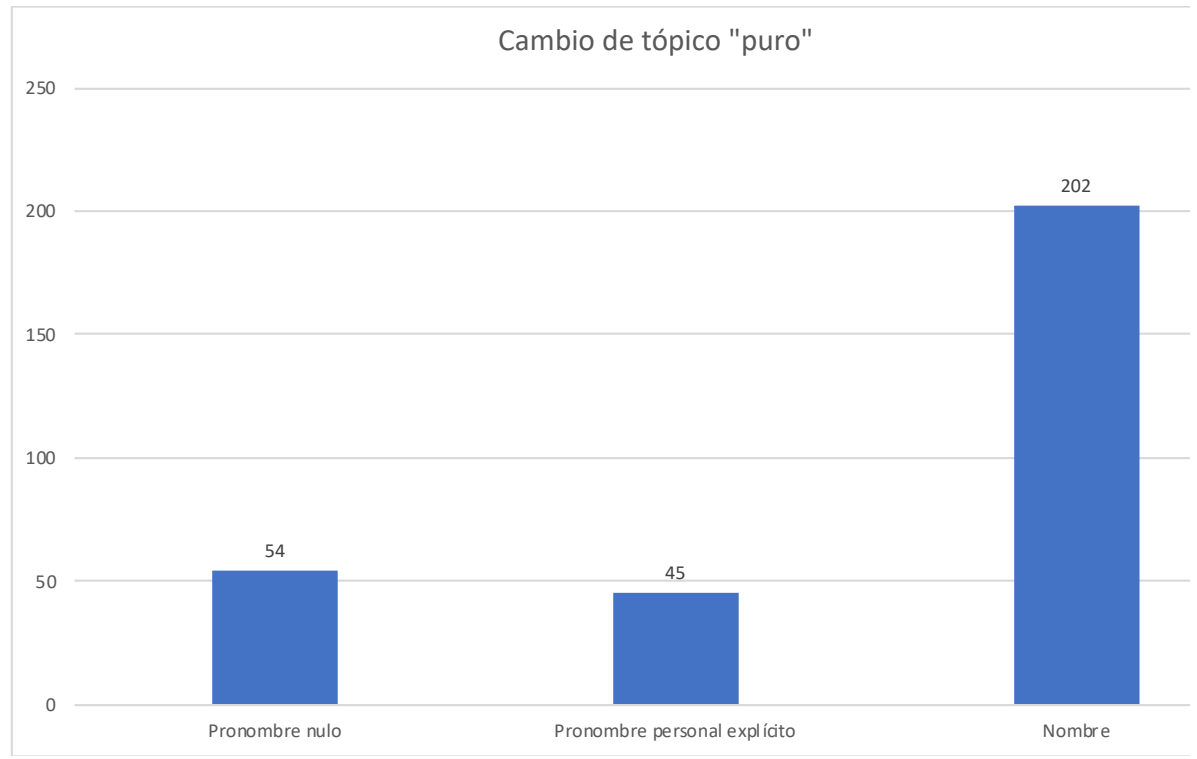

Figura 4. Cambio de tópico «puro»

\section{Conclusión}

En este artículo presentamos los primeros resultados con respecto a las nociones de mantenimiento y cambio de tópico en un corpus de narraciones orales realizadas por aprendices neerlandófonos del español como lengua extranjera. En lo que 
concierne al mantenimiento de tópico, primero, se nota que los aprendices parecen dominar el uso del pronombre nulo, la expresión referencial que, en las escalas de accesibilidad, se correlaciona con los referentes de accesibilidad más alta y que así, son preferibles para mantener la referencia a un tópico. No obstante, también se presentan casos de sobre-explicitación con el empleo de un pronombre personal explícito o el nombre propio, que son expresiones referenciales de accesibilidad más baja. Luego, se observa que se prefiere el nombre propio al marcar un cambio de tópico, al contrario del pronombre personal explícito, que suele presentarse como recurso típico para realizar el cambio. En las escalas de accesibilidad, sin embargo, el nombre propio se enlaza con una accesibilidad baja y así, con la introducción de nuevos referentes. Nuestro estudio se demuestra, pues, que el nombre propio desempeña otros papeles en el discurso que la mera introducción de nuevos referentes. En futuras investigaciones, se estudiará en qué medida los casos de sobre-explicitación pueden explicarse por la influencia de algunos factores que afectan la accesibilidad de un referente, como la distancia entre dos referencias consecutivas o la presencia de competidores.

\section{Bibliografía}

Alonso-Ovalle, L., Fernández-Solera, S., Frazier, L., \& Clifton, Jr., C. 2002. Null vs. overt pronouns and the Topic-Focus articulation in Spanish. Rivista Di Linguistica, 14(2), 151169.

Ariel, M. 1990. Accessing Noun Phrase Antecedents. London and New York: Routledge.

Chafe, W. 1976. Givenness, contrastiveness, definiteness, subjects, topics, and point of view. En C. N. Li (ed.), Subject and Topic. New York: Academic Press, 25-55.

Chafe, W. 1996. Inferring Identifiability and Accessibility. En T. Fretheim \& J. K. Gundel (eds), Reference and referent accessibility. Amsterdam: John Benjamins, 37-46.

Figueras Solanilla, C. 2002. La jerarquía de accesibilidad de las expresiones referenciales en español. Revista Española de Lingüística, 32(1), 53-96.

Gundel, J. K., Hedberg, N. \& Zacharski, R. 1993. Cognitive status and the Form of Referring Expressions in Discourse. Language, 69(2), 274-307.

Lambrecht, K. 1994. Information structure and sentence form - Topic, focus and the mental representations of discourse referents. Cambridge: Cambridge University Press.

Liceras, J. M., \& Díaz, L. 1999. Topic-drop versus pro-drop: null subjects and pronominal subjects in the Spanish L2 of Chinese, English, French, German and Japanese speakers. Second Language Research, 15(1), 1-40.

Lozano, C. 2009. Selective deficits at the syntax-discourse interface: evidence from the CEDEL2 corpus. En Y. Leung, N. Snape, \& M. Sharwood-Smith (eds), Representational Deficits in Second Language Acquisition: Studies in honor of Roger Hawkins. Amsterdam: John Benjamins, 127-166.

Lozano, C. 2016. Pragmatic principles in anaphora resolution at the syntax-discourse interface: advanced learners of Spanish in the CEDEL2 corpus. En M. Alonso Ramos (ed.), Spanish 
Learner Corpus Research: Current Trends and Future Perspectives. Amsterdam: John Benjamins, 236-265.

Lozano, C. 2018. The Development of Anaphora Resolution at the Syntax-Discourse Interface: Pronominal Subjects in Greek Learners of Spanish. Journal of Psycholinguistic Research, 47(2), 411-430.

Lubbers Quesada, M., \& Blackwell, S. E. 2009. The L2 Acquisition of Null and Overt Spanish Subject Pronouns: A pragmatic approach. En J. Collentine et al. (eds), Selected Proceedings of the 11th Hispanic Linguistics Symposium. Somerville: MA: Cascadilla Proceedings Project, 117-130.

Luján, M. 1999. Expresión y omisión del pronombre personal. En V. Demonte \& I. Bosque (eds), Gramatica descriptiva de la lengua española, 1275-1316.

O'Donnell, M. 2008. The UAM CorpusTool: software for corpus annotation and exploration. En Proceedings of the XXVI Congreso de AESLA. Almería.

Perales, S., \& Portillo, M. R. 2007. Sobre las propiedades referenciales de los sujetos nulos y pronominales del español oral y escrito. En Las destrezas orales en la enseñanza del español L2-LE: XVII Congreso Internacional de la Asociación del Español como lengua extranjera (ASELE), 2, 889-900.

Porto Dapena, Á. 1986. Los pronombres. Madrid: EDI-6.

Prince, E. F. 1981. Toward a Taxonomy of Given-New Information. En P. Cole (ed.), Radical Pragmatics. New York: Academic Press, 223-256.

Rothman, J. 2009. Pragmatic deficits with syntactic consequences?: L2 pronominal subjects and the syntax-pragmatic interface. Journal of Pragmatics, 41, 951-973. 